$$
\text { DOE/ER/45/20 - T B }
$$

\title{
A Frontier Molecular Orbital Determination of the Active Sites on Dispersed Metal Catalysts
}

\author{
Robert L. Augustine and Konstantinos M. Lahanas \\ Department of Chemistry, Seton Hall University, South Orange NJ 07079 DE93 002764
}

\begin{abstract}
An angular overlap calculation has been used to determine the s, $\mathbf{p}$ and $d$ orbital energy levels of the different types of surface sites present on a dispersed metal catalysts. The basis for these calculations is the reported finding that a large number of catalyzed reactions take place on single atom active sites on the metal surface. Thus, these sites can be considered as surface complexes made up of the central active atom surrounded by near-neighbor metal atom "ligands" with localized surface orbitals perturbed only by these "ligands". These "complexes" are based on a twelve coordinate species with the "ligands" attached to the $t_{2 g}$ orbitals and the coordinate axes coincident with the direction of the $e_{g}$ orbitals on the central atom. These data can permit a Frontier Molecular Orbital treatment of specific site activities as long as the surface orbital availability for overlap with adsorbed substrates is considered along with its energy value and symmetry.
\end{abstract}

\section{Introduction}

An understanding of the nature of the surface orbitals of a catalytically active site is essential for determining the molecular processes which can take place on such sites. This type of information can provide a means of correlating a given type of catalyst:substrate interaction with the formation of a specific product, data which could eventually lead to the ability to design new catalyzed processes from first principles. It would seem that Frontier Molecular Orbital considerations which have been so useful in organic synthesis [1] could also prove beneficial in this instance. However, with metal catalysts one is faced with the problem of dealing with complex three dimensional species which do not have discrete electronic energy levels. This difficulty has been approached most recently by thinking about groups of electronic levels rather than individual ones and utilizing the calculated density of states (DOS) in studying the nature of catalyst:substrate interactions [2 - 8]. The DOS is the relative number of states in a given energy level interval and corresponds, roughly, to the electron levels of a monoatomic species. The surface DOS have been calculated for the 111 and 100 faces of a number of metals [2 - 9]. Interestingly, these surface bands are rather narrow indicating a reasonable degree of localization for the atomic orbitals on the metal surface. Changes in the calculated DOS curves brought about by placing substrate molecules at various positions on these planar surfaces has led to the determination of the most favorable location for adsorption of these substrates on the metal surface under consideration [2 - 8]. Another approach to the development of a quantum chemical understanding of catalysis [9 - 11] has incorporated the symmetry aspects of chemisorption [10] and has been used to describe the effect of surface unsaturation and the presence of additives on the chemisorption process [11, 12] as well as FMO considerations of these surface:substrate interactions $[6,9,12]$. The results of these calculations agree quite well with available experimental data.

However, data have been reported which show that while reactions involving $\mathbf{C}-\mathbf{C}$ bond hydrogenolysis or hydrocarbon isomerizations occur on ensembles of atoms on the faces of the

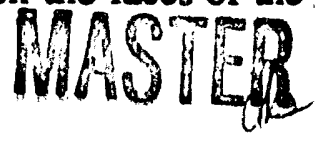

1 ESTRIBUTION OF THIS DOCUMENT IS UNLIMITED 
metal crystallites present on supported metal catalysts, most other catalytic reactions, particularly those involving $\mathrm{C}-\mathrm{H}$ bond breaking or formation, take place on single atom active sites [13, 14], probably the more coordinately unsaturated comer atoms [15, 16]. Thus, while the DOS and other types of calculations have been successful in determining the adsorption characteristics of the 111 and 100 metal planes, extension of this approach to the determination of the adsorption and reaction characteristics of the single atom active sites present as edge and comer atoms resulting from the intersection of these planes is not straightforward.

A knowledge of edge and comer atom activity, however, is essential for the detailed understanding of most present catalytic processes and the development of new ones. One way of simplifying this problem is to invoke the Isoglobal Analogy [17] whirh states that fragments of a metal particle can be considered as approximations of the catalytically active surface sites. An obvious example is the use of small metal clusters as analogs for the study of catalytic activity. Molecular orbital calculations on small metal clusters have provided complete electronic data for these species [18 - 20] but it is not clear how this information can be extrapolated to an understanding of the adsorption characteristics of the corner atoms on metal catalysts. Cluster valence molecular orbitals (CVMO) have also been calculated for a number of different types of metal clusters to provide the overall valence electron availability for the cluster [21, 22]. By investigating a number of cluster shapes the CVMO was determined for a variety of different types of surface atoms. While these data provided the $s, p$, and $d$ electronic character of each type of site, the energy levels for these various orbitals were not determined [22]. This work, however, did serve to emphasize the complexity of the surfaces present on a dispersed metal catalyst.

Thus, with the catalytically active site for most reactions being a single surface atom $[13,14]$ and the Isoglobal Analogy [17] stating that such sites can be thought of as discrete entities, it would seem reasonable, as a simplifying assumption, to consider the surface of a metal particle as being made up of a number of different types of "surface complexes" composed of a central metal active site atom surrounded by its nearest neighbor "ligands" [24]. To complete this "surface complex" analogy it must also be assumed that the surface orbitals of this active site are localized and that the active site atomic orbitals are perturbed only by interaction with the nearest neighbor, "ligand", atoms. The degree of surface electron localization found by the DOS calculations lends support to this assumption [2 - 8].

\section{Discussion}

Several attempts have been made to relate possible reactions on specific surface sites with those promoted by catalytically active monoatomic complexes [25 - 29]. While such comparisons have led to a somewhat better understanding of the problems associated with the presence on the metal surface of different types of active sites, such direct comparisons are generally invalid mechanistically. Almost all of the monoatomic complexes used for these comparisons have an octahedral configuration, yet, because of the orientation of the $t_{2 \mathrm{~g}}$ orbitals involved in the bonding with the nearest neighbor atoms in fcc metals [23], an octahedral "surfare complex" made up of a central atom and nearest neighbor "ligands" cannot exisi. Instead of the commonly used octahedral orientation, the "surface complexes" on the catalyst surface are based on the twelve coordinate species shown in Fig. 1. Each of the different types of surface sites are derived from this parent species by removing varying numbers of the "ligands".

Table 1 lists the different types of surface atoms which can be present on crystallites of fcc metals, the lattice orientation of most catalytically active metals. Each of these "surface complexes" are depicted in Fig. 2. There are atoms on the two low Miller index faces, the 111 (site A) and 100 (site B), those commonly used in theoretical calculations. There are also 
four possible edge sites made up by the intersection of these planes. Both the 111-111 and 111-100 edges can result from plane intersections of $120^{\circ}$ (wide) or $60^{\circ}$ (narrow). For reaction purposes the 110 face is best described as a series of narrow 111-111 edges in close proximity to each other. Since in the fcc metal lattice the nearest neighbor atoms are bonded by $t_{2 g} \mathrm{~d}$ orbitals [23], the $90^{\circ}$ intersection is not permitted so the cubic 100-100 edge does not exist. The $90^{\circ}$ angles associnted with the $e_{8}$ orbitals are found in the orientations of the next nearest neighbor atoms in the crystal lattice [23]. In addition to these face and edge atoms, there are at least seven different types of corner atoms ranging from the cubooctahedral comer (site G) with six nearest neighbors to the tetrahedral comer (adatom on a 111 face) (site M) with only three nearest neighbors. The tetrahedral corner is the result of the $60^{\circ}$ intersection of the three planes. When one of these planes intersects the others at $120^{\circ}$ a wide corner (site $J$ ) is produced. Further modifications to these and the octahedral corner (adatom on a 100 face) (site K) can give the other nixixed 111 and 100 comers listed in Table 1 and shown in Fig. 2. The specific near neighbor "ligands" present on the central atom active site for each of these "complexes" are also listed in Table 1 so an indication of a specific site composition can be ottained using these lata in conjunction with the drawings in Fig. 1.

\section{Angular Overlap Model}

With the assumption being made that the catalytically active sites on a metal particle can be treated as "surface complexes" it should be possible to extend the analogy and calculate the orbital energy levels for each site using classical inorganic techniques. That approach considered to be the most useful in the present instance is the angular overlap model (AOM) $[30,31]$ which is sufficiently general to be applicable to the wide range of sites shown in Fig. 2. The AOM is a simple approximation of the full MO model which still contains all of the important characteristics of the metal-ligand $(M-L)$ interactions in complexes. The primary difference between these two models is the simplifying assumption in the AOM that the energy values for the entire complex are considered as the sum of the energies of the individual $\mathrm{M}-\mathrm{L}$ interactions. The energy of each $\mathrm{M}-\mathrm{L}$ interaction is proportional to the square of the overlap integral between orbitals on the $\mathrm{M}$ and $\mathrm{L}$ atoms. This overlap can be broken down into two integrals, one composed of the radial components and the other comprised of the angular components of the overlapping atomic orbitals. AOM is used to determine the angular dependance of the overlap integral for each type of orbital. 
Table 1

Types Of Surface Atoms On fcc Metals

\begin{tabular}{|c|c|c|c|}
\hline $\begin{array}{c}\text { Site } \\
\text { Description }\end{array}$ & $\begin{array}{l}\text { \# Nearest } \\
\text { Neighbors }\end{array}$ & Ligands & Desig'n. \\
\hline $\begin{array}{l}111 \\
\text { (face) }\end{array}$ & 9 & $1,2,3,4,5,6,7,8,9$ & $\bar{A}$ \\
\hline $\begin{array}{l}100 \\
\text { (face) }\end{array}$ & 8 & $1,2,3,5,6,7,8,11$ & B \\
\hline $\begin{array}{l}111-100 \\
\text { (wide edge) }\end{array}$ & 7 & $1,2,3,5,6,7,8$ & C \\
\hline $\begin{array}{l}111-111 \\
\text { (wide edge) }\end{array}$ & 7 & $1,2,3,4,5,6,7$ & D \\
\hline $\begin{array}{l}111-111 \\
\text { (narrow edge) }\end{array}$ & 6 & $2,3,5,6,7,8$ & E \\
\hline $\begin{array}{l}111-100 \\
\text { (narrow edge) }\end{array}$ & 5 & $2,4,5,6,7$ & $\mathbf{F}$ \\
\hline $\begin{array}{l}111-111-100 \\
\text { (cubooctahedral corner) }\end{array}$ & 6 & $1,2,3,6,7,8$ & $G$ \\
\hline $\begin{array}{l}111-111-100-100 \\
\text { (comer) }\end{array}$ & 5 & $1,2,3,7,8$ & $\mathbf{H}$ \\
\hline $\begin{array}{l}111-111-111 \\
\text { (wider comer) }\end{array}$ & 5 & $1,2,3,6,7$ & I \\
\hline $\begin{array}{l}111-111-111 \\
\text { (wide comer) }\end{array}$ & 4 & $1,3,7,3$ & $\mathbf{J}$ \\
\hline $\begin{array}{l}111-111-111-111 \\
\text { (octahedral corner) }\end{array}$ & 4 & $2,3,6,7$ & $\mathbf{K}$ \\
\hline $\begin{array}{l}111-111-100 \\
\text { (narrow corner) }\end{array}$ & 3 & $1,3,8$ & $\mathbf{L}$ \\
\hline $\begin{array}{l}111-111-111 \\
\text { (tetrahedral corner) }\end{array}$ & 3 & $1,2,3$ & $\mathbf{M}$ \\
\hline
\end{tabular}

As depicted in Fig. 1. 

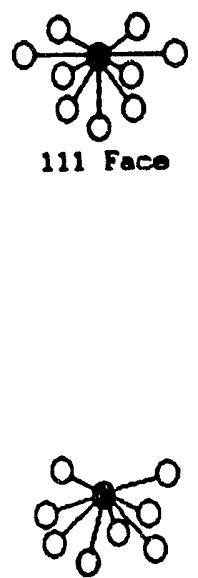

100 Face

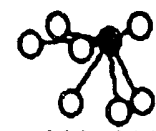

111-111

Vide Edge

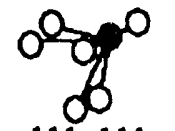

$111-111$
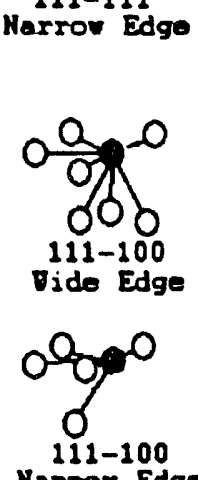

Narrov Edge

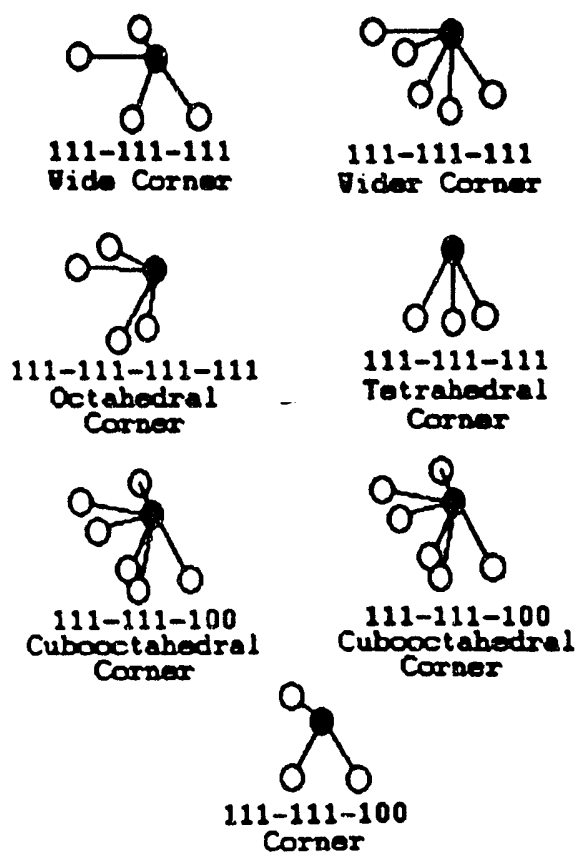

Fig. 2. Diagrams of the various types of atoms present on the surface of an fcc metal.

The spherical coordinates which are required for AOM calculations [30, 31] were determined by placing the $x, y$, and $z$ axes coincident with the direction of the $e_{s}$ orbitals on the central atom as depicted in Fig. 1. These axes were thus fixed in a constant orientation for all of the "surface complexes". For example, the 111 face atom (site A) is composed of the central atom surrounded by the nearest neighbors 1 through 9 as depicted in Fig. 1. The $x, y$, and $z$ axes each intersect the central atom at an angle of $30^{\circ}$ from the plane of the six ligands, 4 -9. This is the angle of protrusion of localized $e_{g}$ orbitals from an atom on a 111 face [32]. The tetrahedral comer is comprised of the central atom with only the 1,2, and 3 ligands. The $x, y$, and $z$ axes, though, are still in the same orientation with respect to the central atom as in the 111 face site.

To determine the orbital energy levels for a site the spherical coordinates for each ligand were inserted into the appropriate angular overlap matrix developed by Shäffer [33] to obtain the individual $p$ and $d$ orbital overlap integrals between the central atom and the ligand. These are then squared and summed to give the overlap energy for the specific ligand. This process is repeated for each ligand and the results added to give the energies of each p orbital in terms of $e_{p \sigma}$ and $e_{p \pi}$ and each $d$ orbital in terms of $e_{d \sigma}, e_{d y}$ and $e_{d \delta}$, which are factors in the radial component of the $M-L$ overlap integrals [30, 30]. Since s orbitals are symmetrical there is no angular component associated with s orbital overlap so the s overlap integrals are expressed in terms of $e_{s \sigma}$ multiplied by the number of "ligands" associated with the "surface complex". The energy terms for the s, p and d orbitals on the 111 face atom (Type A) and the tetrahedral corner (Type M) expressed in this way are listed in Table 2.

To this point the solution is generic and can be applied to any metal with an fcc crystal orientation. The differences between these metals lie in the values of the $s, p$, and $d$ overlap integrals for each metal. In the classic AOM procedure [31], the solution for these parameters 
Table 2

Angular Components of the d Orbital Overlap Energies for the 111 Face (Site A) and Tetrahedral Corner (Site M) Surface Sites

\begin{tabular}{|c|c|c|c|}
\hline $\begin{array}{c}\text { Site } \\
\text { Desig'n }\end{array}$ & Electron & Orbital & Angular Component \\
\hline \multirow[t]{3}{*}{ A } & $4 \mathrm{~s}$ & $\mathbf{s}$ & $9.000 * e_{8 \sigma}$ \\
\hline & $4 p$ & $\begin{array}{l}x \\
y \\
z\end{array}$ & $\begin{array}{l}3.000 * e_{p \sigma}+6.000 * e_{p r} \\
3.000 * e_{p \sigma}+6.000 * e_{p n} \\
3.000 * e_{p \sigma}+6.000 * e_{p n}\end{array}$ \\
\hline & $3 d$ & $\begin{array}{c}z^{2} \\
y z \\
x z \\
x y \\
x^{2}-y^{2}\end{array}$ & $\begin{array}{l}1.125 * e_{\mathrm{d} \sigma}+4.500 * e_{\mathrm{d} \pi}+3.375 * e_{d \delta} \\
2.250 * e_{\mathrm{d} \sigma}+3.000 * e_{\mathrm{d} \pi}+3.750 * e_{d \delta} \\
2.250 * e_{\mathrm{d} \sigma}+3.000 * e_{\mathrm{d} \pi}+3.750 * e_{d \delta} \\
2.250 * e_{\mathrm{d} \sigma}+3.000 * e_{\mathrm{d} \pi}+3.750 * e_{d \delta} \\
1.125 * e_{\mathrm{d} \sigma}+4.500 * e_{\mathrm{d} \pi}+3.375 * e_{d \delta} \delta\end{array}$ \\
\hline \multirow[t]{3}{*}{$\mathbf{M}$} & $4 s$ & $\mathbf{s}$ & $3.000 * e_{s \sigma}$ \\
\hline & $4 p$ & $\begin{array}{l}x \\
y \\
z\end{array}$ & $\begin{array}{l}1.000 * e_{p \sigma}+2.000 * e_{p \pi} \\
1.000 * e_{p \sigma}+2.000 * e_{p \pi} \\
1.000 * e_{p \sigma}+2.000 * e_{p \pi}\end{array}$ \\
\hline & $3 d$ & $\begin{array}{l}z^{2} \\
y z \\
x z \\
x y \\
x^{2}-y^{2}\end{array}$ & $\begin{array}{l}0.375 * e_{d \sigma}+1.500 * e_{d \pi}+1.125 * e_{d \delta} \\
0.750 * e_{d \sigma}+1.000 * e_{d \pi}+1.250 * e_{d \delta} \\
0.750 * e_{d \sigma}+1.000 * e_{d \pi}+1.250 * e_{d \delta} \\
0.750 * e_{d \sigma}+1.000 * e_{d \pi}+1.250 * e_{d \delta} \\
0.375 * e_{d \sigma}+1.500 * e_{d \pi}+1.125 * e_{d \delta}\end{array}$ \\
\hline
\end{tabular}

${ }^{\text {a As designated in Table } 1 .}$

is based on the assumption that the electron densities on the central atom and the ligand were different but in the present case, the central atom and "ligand" atom are of the same species so this classic procedure cannot be used. Instead, the values of $e_{\mathrm{s} \sigma}, e_{\mathrm{p} \sigma}, \mathrm{e}_{\mathrm{p} n}, \mathrm{e}_{\mathrm{d} \sigma}$, $e_{\mathrm{d} n}$ and $e_{\mathrm{d} \sigma}$ were determined for each metal by an EHMO calculation on the diatomic $\mathbf{M}-\mathbf{M}$ with the overlap integrals calculated from Slater type orbitals using published Huckel parameters [3] for the surface metal atoms and the bulk interatomic distance of the metal. The various orbital energy levels for each type of surface site were then calculated with the results for Pd depicted graphically in Fig. 3. Comparable data for the other catalytically active metals have also been obtained. 


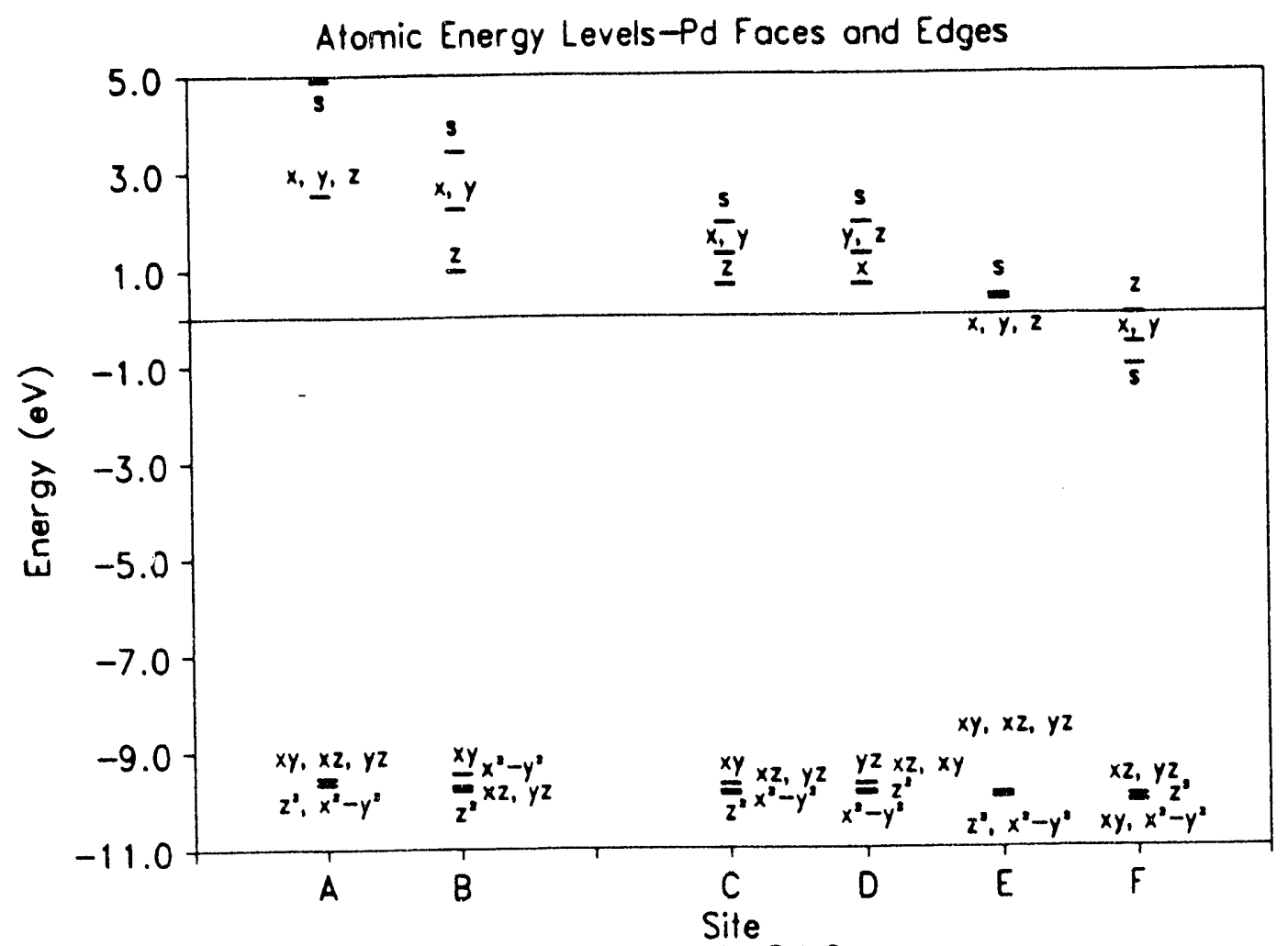

Atomic Energy Levels-Pd Corners

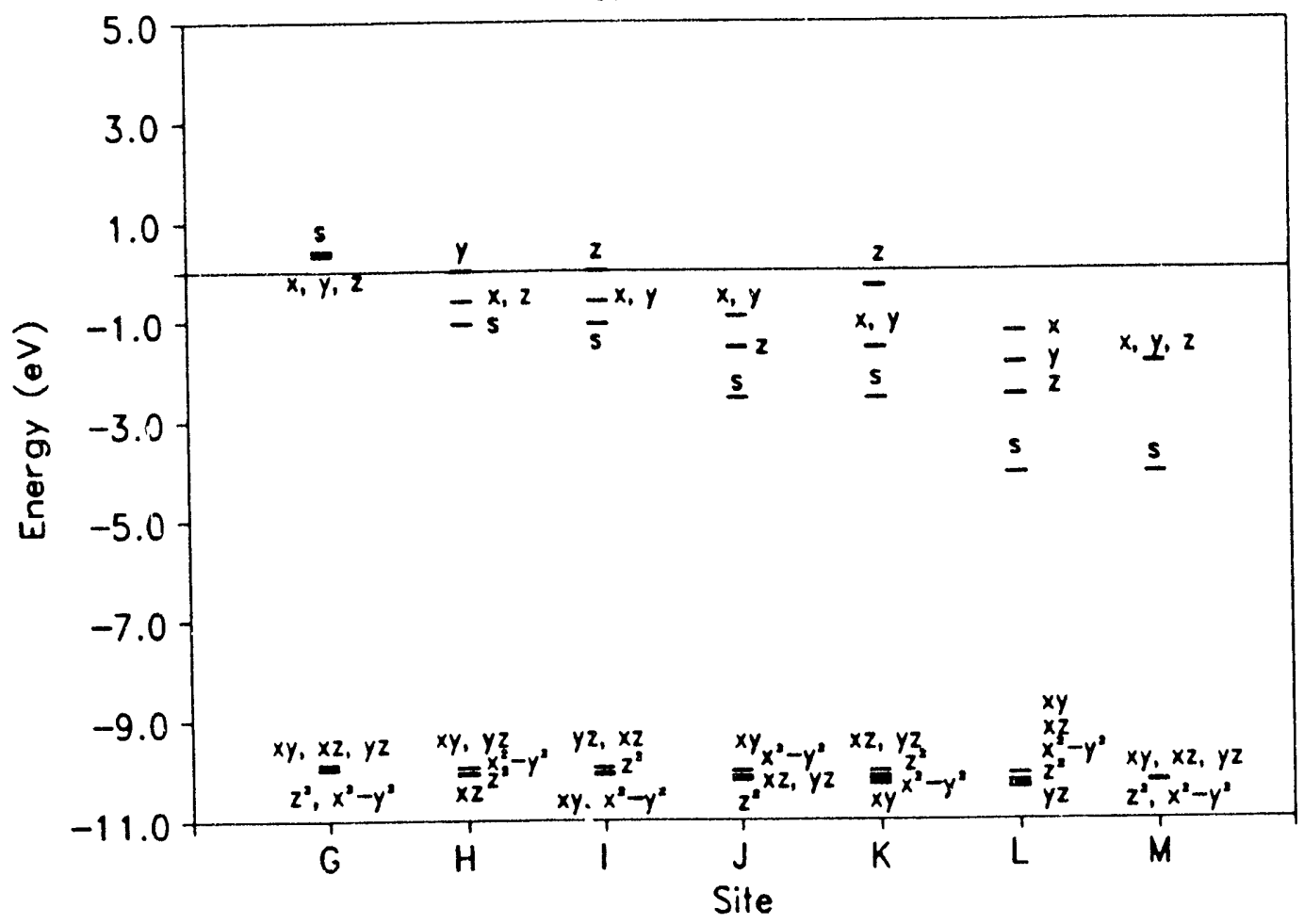

Fig. 3. Electron energy levels for various types of Pd surface atoms. Horizontal label equivalence indicates orbital degeneracy. The vertical label orientation depicts relative energy values. 
Direct comparison of these data
with existing results is not straightforward since, as mentioned previously, most of the reported computations have been made on the 111 and 100 faces [2 - 12]. A clear correlation between the AOM results and the EHMO DOS data may not be forthcoming since the AOM calculations are based on assumptions which are not involved in the more rigorous EHMO calculations. Probably the most significant of these is that in the AOM only the nearest neighbor "ligand" atoms are considered in the perturbation of the electronic character of the central atom while in DOS and similar EHMO calculations all atoms are involved. These AOM data, however, are more easily obtained and they can be useful in developing a viajle correlation between specific site type and activity. For instance, these results indicate that the more coordinately unsaturated corner atoms have lower orbital energy levels than the face atoms so these corner atoms are expected to be more reactive. These data can also be used for a Frontier Molecular Orbital (FMO) development of specific site reactivity. For this to be effective, though, it must be kept in mind that these surface sites are rigid and have definite geometric and steric relationships between the active sites and the nearby surface atoms, be they "ligands" or not. Thus, some of the surface orbitals may be oriented in such a way that they are sterically not available for chemisorption even if the energy and symmetry are correct. It is essential, then, that these steric constraints to maximum substrate interaction be determined before sing this FMO approach. This is facilitated by recognizing that according to
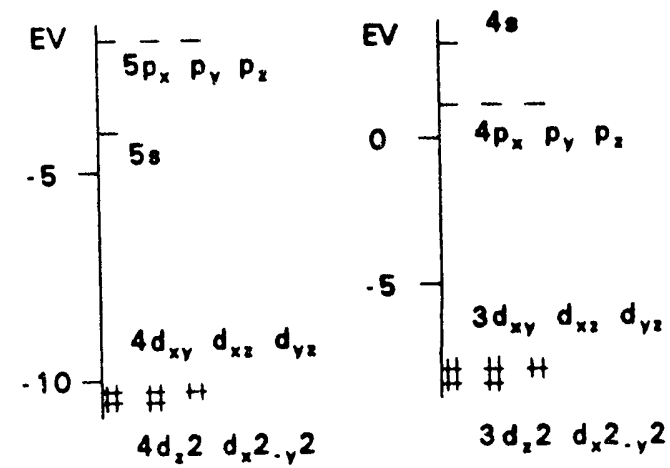

$\mathbf{a}$

b
Fig. 5. Orbital energies for: a) $\mathrm{Pd}$ tetrahedral corner atom (Site M); b) Ni 111 face atom (Site A). the numbering scheme and axes orientations shown in Fig. 1, ligands 1, 5, 8, and 11 are attached to the $d_{x y}$ orbital, $2,4,7$, and 12 to the $d_{y z}$, and $3,6,9$, and 10 to the $d_{x z}$. Once the geometry of the "ligands" on thesc sites is recognized, the nature and angle of protrusion of the remaining $t_{2 g}$ lobes follows. The $x, y$, and $z$ axes correspond to the orientation of the deg and p orbitals. Care should be taken to avoid using any of the surface lobes which project close to the surface for any substrate interactions.

For example, it has been calculated that $\mathrm{CO}$ adsorption on $\mathrm{Ni} 100$ face atoms takes place preferentially on top of the surface atoms [3]. The data in Fig. 4 represent the electron energy levels for an atom on a $\mathrm{Ni} 100$ face. The LUMO $4 \mathrm{p}_{2}$ orbital is available to accept the $5 \sigma$ electrons 
of the $\mathrm{CO}$ molecule but the HOMO $\mathrm{d}_{\mathrm{xy}}$ orbitals are in the 100 plane and used for bonding the central atom to ligands 1,5 , 8 , and 11. The next lower orbital, $d \times 2-y^{2}$, corresponding to the $x$ and $y$ axes, also lies in the 100 plane and is, thus, also unavailable for surface bonding. Backbonding to the $2 \pi$ orbitals of the $\mathrm{CO}$ can only be accomplished using the degenerate $d_{x z}$ or $d_{y z}$ orbitals which protrude from the surface at a $45^{\circ}$ angle [32] and have the proper symmetry.

The single atom active site responsible for the hydrogenation of double bonds, however, must have sufficient coordinative unsaturation to chemisorb both the $\mathrm{H}_{2}$ molecule and the alkene on the same atom. Such a site could be the tetrahedral corner (Site $M$, Fig. 2). This site is composed of the central atom and "ligands" 1, 2 and 3 as depicted in Fig. 1. The surface orbital energies for a Pd atom at a tetrahedral corner are shown in Fig. Sa. The filled $\mathrm{d}$ ortitals not involved in $\mathbf{M}-\mathbf{M}$ bonding are directed toward the other ligand positions. As depicted in Fig. 6, $\mathrm{H}_{2}$ chemisorption can take place on this site by $\sigma$ donation froin the H-H bond to the LUMO $4 \mathrm{~s}$ orbital. Backbonding to the $\sigma^{*}$ orbitals can occur using the HOMO $\mathrm{d}_{x z}$ orbital lobes directed toward positions 9 and 10 . Double bond adsorption on the resulting $\mathrm{M}-\mathrm{H}_{2}$ species can take place through alkene $\pi$ donation to the now LUMO py orbital along with back bonding to the $\pi^{*}$ orbitals using the HOMO $d_{x y}$ orbital lobes directed toward positions 8 and 11 . The catalytic cycle is then completed by hydrogen insertion to give a hydrido metalalkyl which then undergoes reductive elimination to produce the alkane and regenerate the active site. Similar considerations can be used to rationalize the reactivity of the other more coordinately unsaturated sites.

Even though these AOM data were derived using single atom active sites, one can envision a combination of these sites to form an active "ensemble" with localized electrons on each atom which can be used to develop an understanding of the reactivity of the multiple atom site. As a case in point, calculations of hydrogen adsorption on a $\mathrm{Ni} 111$ face indicate that the three-fold adsorption site pictured in Figure 7a is favored [2]. This can be understood using the localized electron levels on the $111 \mathrm{Ni}$ atoms as depicted in Fig. 5b. As shown in Fig. $\mathrm{Fb}$, the LUMO $\mathrm{p}_{\mathrm{z}}$ orbital on atom 3 protrudes from the surface at an angle of $30^{\circ}$ [32] and can, thus, overlap the $\sigma$ bond of the $\mathrm{H}-\mathrm{H}$ molecule. The HOMO $d_{x z}$ lobes on atoms 1 and 2 protrude from the surface at a $60^{\circ}$ angle [32] and are properly oriented for 
interaction with the $\sigma^{*}$ orbitals. This is a three atom analog of the single atom adsorption depicted in Fig. 6.

It appears, then, that AOM calculations on single atom active site "surface complexes" on a metal catalyst can provide data concerning the localized surface electron energy levels which are useful in understanding the adsorption and reaction characteristics of the various types of sites present on dispersed metal catalysts. The primary factor in the use of these data is that the availability of the orbitals for overlap with adsorbing substrates must be considered along with their energy and symmetry.

\section{Acknowledgement}

This research was supported by Grant DE-FG02-84ER45120 from the U.S. Department of Energy, Office of Basic Energy Science. We also wish to express our appreciation to Prof. Roald Hoffmann for the comments and suggestions made during the preparation of this manuscript.

\section{References}

1. R. B. Woodward and R. Hoffmann, Conservation of Orbital Symmetry Academic Press, NY, 1970.

2. J. -Y. Saillard and R. Hoffmann, J. Am. Chem. Soc., 106, 2006 (1984).

3. S. -S. Sung and R. Hoffmann, J. Am. Chem. Soc., 107, 578 (1985).

4. J. Silvestre and R. Hoffmann, Langmuir, 1, 621 (1985).

5. J. Li, B. Schioett, R. Hoffmann and D. Proserpio, J. Phys. Chem., 94, 1554 (1990).

6. M. C. Zonnevylle, R. Hoffmann, P. J. Van den Hoek and R. A. Van Santen, Surf. Sci., 223, 233 (1989).

7. C. Zheng, Y. Apeloig and R. Hoffmann, J. Am. Chem. Soc., 110, 749 (1988).

8. M. C. Zonnevylle and R. Hoffmann, Langmuir, 3, 452 (1987).

9. R. A. van Santen, Prog. Surf. Sci., 25, 253 (1987).

10. R. A. van Santen, J. Mol. Structure, 173, 157 (1988).

11. R. A. van Santen, J. Mol. Catal., 54, 288 (1989).

12. R. A. van Santen, J. Chem. Soc., Faraday Trans. I, 83, 1915 (1987).

13. J. H. Sinfelt, J. L. Carter and D. J. C. Yates, J. Catal., 24, 283 (1972).

14. P. S. Kirlin and B. C. Gates, Nature, 325, 38 (1987).

15. R. L. Augustine and M. M. Thompson, J. Org. Chem., 52, 1911 (1987).

16. R. K. Herz,W. D. Gillespie, E. E. Petersenand G. A. Somorjai, J. Catal., 67, 371 (1981).

17. R. Hoffmann, Angew. Chem., 21, 711 (1982).

18. J. -F. Halet, R. Hoffmann and J. -Y. Saillard, Inorg. Chem., 24, 1695 (1985).

19. G. F. Holland,D. E. Ellis and W. C. Trogler, J. Chem. Phys., 83, 3507 (1985). 
20. N. C. Datta and B. Ser, J. Chem. Soc., Faraday Trans. 2, 82, 977 (1986).

21. J. W. Lauher, J. Am. Chem. Soc., 100, 5305 (1978).

22. J. W. Lauher, J. Am. Chem. Soc., 101, 2604 (1979).

23. J. B. Goodenough, Magnetism and the Chemical Bond, Interscience, NY, 1963.

24. W. M. H. Sachtier, Ultramicroscopy, 20, 135 (1986).

25. S. Siegel, J. Outlaw, Jr. and N. Garti, J. Catal., S2, 102 (1978).

26. R. L. Augustine and J. F. Van Peppen, Ann. N.Y. Acad. Sci., 172, 244 (1970).

27. R. L. Augustine and R. W. Wamer, J. Catal., 80, 358 (1983).

28. M. J. Ledoux, J. Catal., 70, 375 (1981).

29. G. V. Smith, A. Molnar, M. M. Khan, D. Ostgard and N Yoshida, J. Catal., 98,502 (1986).

30. J. K. Burdett, Adv. Inorg. and Radiochem., 21, 113 (1978).

31. R. S. Drago, Physical Methods in Inorganic Chemistry, Saunders, 1977, pp 396-406.

32. G. C. Bond, Disc. Faraday Soc., 41, 200 (1966): Surf. Sci. , 18, 11 (1969).

33. C. E. Schäffer, Pure and Applied Chem., 24, 361 (1970).

\section{DISCLAIMER}

This report was prepared as an account of work sponsored by an agency of the United States Government. Neither the United States Government nor any agency thereof, nor any of their employees, makes any warranty, express or implied, or assumes any legal liability or responsibility for the accuracy, completeness, or usefulness of any informaticin, apparatus, product, or process disclosed, or represents that its use would not infringe privately owned rights. Reference herein to any specific commercial product, process, or service by trade name, trademark, manufacturer, or otherwise does not necessarily constitute or imply its endorsement, recommendation, or favoring by the United States Government or any agency thereof. The views and opinions of authors expressed herein do not necessarily state or refiect those of the United States Government or any agency thereof. 

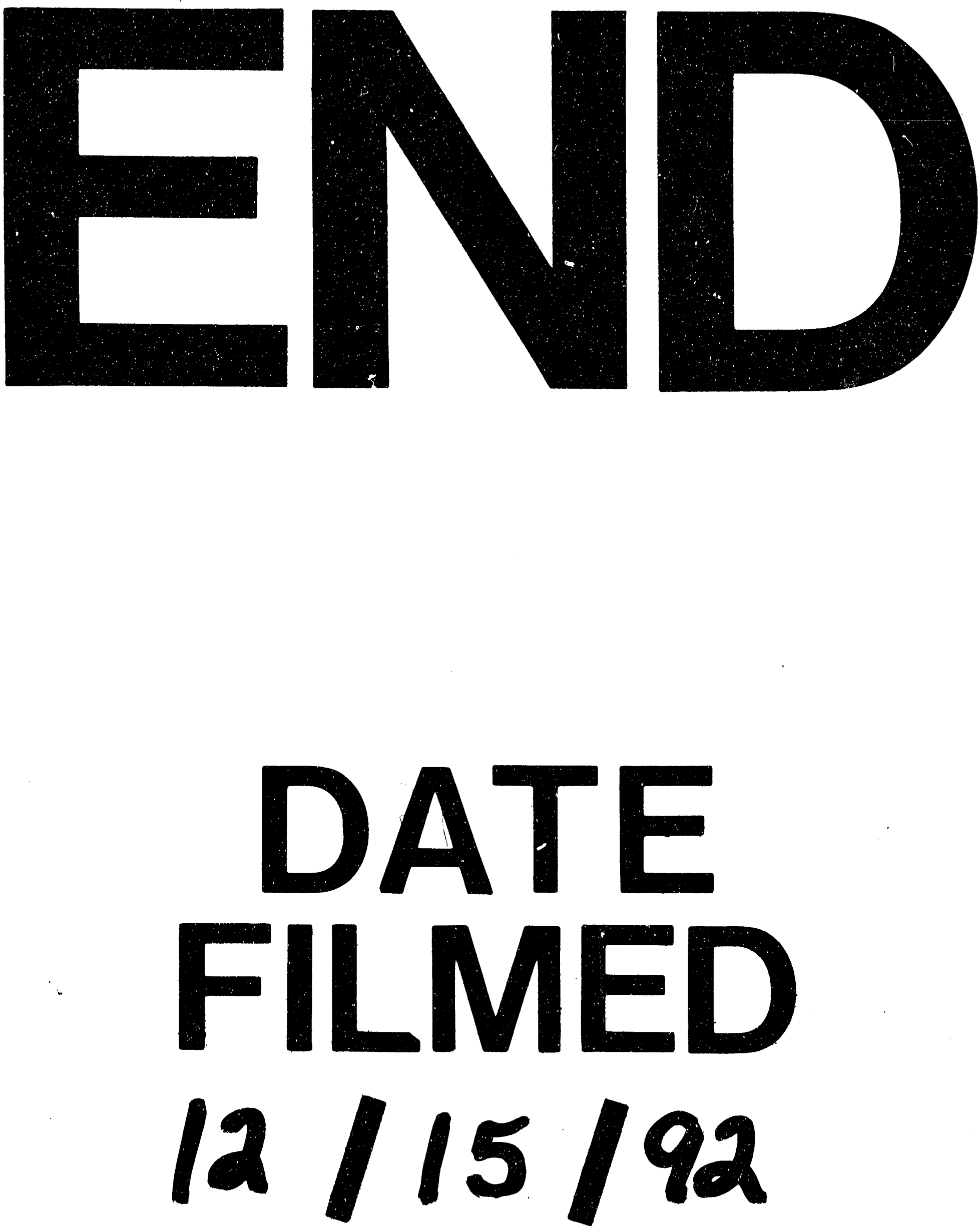